\title{
The Electric Control System for Part Handling Device
}

\author{
GuangZhen Cheng ${ }^{1, a}$, Xiang PEl ${ }^{2, \text { b }}$, Qian Zhou ${ }^{1, c}$, ShiYang Wei ${ }^{1, d}$ \\ ${ }^{1}$ Huzhou Teachers College, Huzhou, 313000, P.R. China \\ 2 Henan Polytechnic Institutes, Nanyang 473000, P.R. China

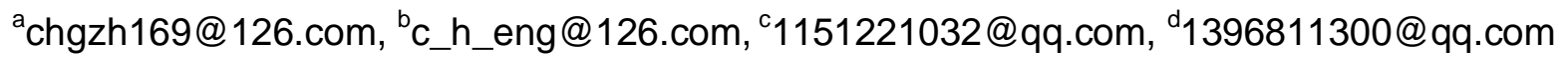

Keywords: Electric Control; Handling Device; Ladder Diagram, PLC Program

Abstract. Design content includes the choice of controller, design of external wiring, design of PLC programming. According to the movement process, write PLC program. The program has four components, the initialization program, the manual operation program, the automatic return to the origin program, and automatic working program. Simulating and debugging the program. The work piece on stepping conveyor was moved to the processing machine tool worktable. The device can work in succession, improve working environment, reduce labor intensity, and improve the level of automation.

\section{Introduction}

This paper introduced an electric control system for part handling device. The handling device was composed of a frame body, a moving device, a horizontal moving oil cylinder YG1, an upper and a lower movable oil cylinder YG2, a clamping oil cylinder YG3, a conveyor, a working table and a stroke switch. The oil cylinder YG1 is fixed on the frame body, and the oil cylinder YG1 piston rod is fixedly connected with the mobile device, which drives the moving device to realize horizontal movement along the guide rail of the mobile device, and the upper and lower moving oil cylinder YG2 is fixed on the moving device. The oil cylinder YG2 piston rod is fixedly connected with the clamping oil cylinder YG3, the oil cylinder YG2 piston rod can be moved up and down to move up and down, and the clamping oil cylinder YG3 drives the clamping block to realize the clamping and loosening. When the automatic modes operate, rely on the travel switch to achieve the action conversion. Travels switch installation location accurate, handling device motion position accurately. This design were installed upper limit position switch SQ0, down limit switch SQ1, left limit travel switch SQ2, right limit travel switch SQ3. The limit switch SQ0, SQ1 is relatively fixed with mobile device. The limit switch SQ2, SQ3 is relatively fixed with frame. The parts detecting switch SQ4 is fixed on the frame body, to ensure that there is no object on the working table, only then the feeding mechanism is allowed to drop and the parts is put into the working table. The structure of the handling device was shown in Fig.1.

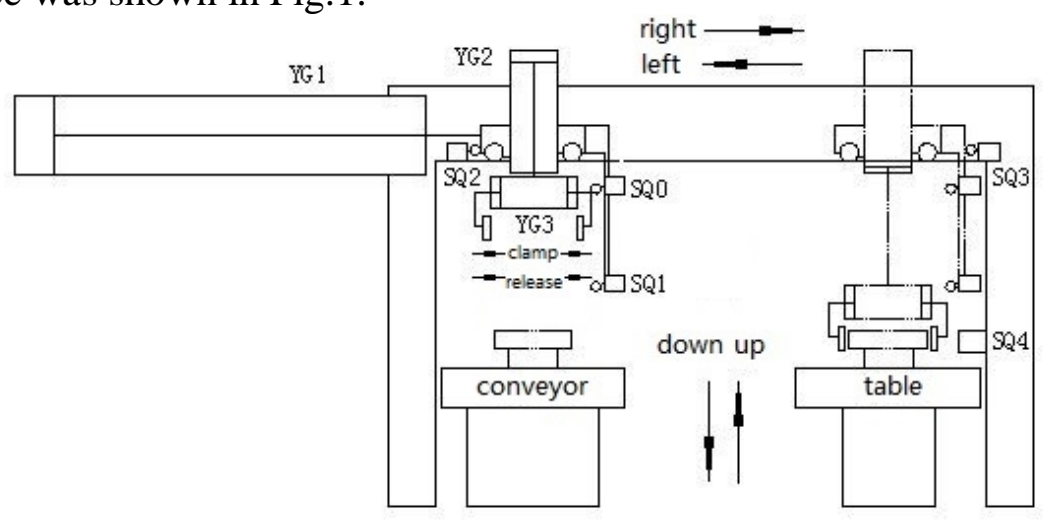

Fig.1 Structure schematic diagram 


\section{PLC Control Program}

PLC uses Mitsubishi Co's programmable logic controller, the model was 48MR - FX2N. The control panel components layout was shown in Fig.2. The PLC control wiring diagram was shown in Fig.3. The operating mode was selected with rotary switch, when the rotary switch SA were located SA-1, SA-2, SA-3, SA-4, SA-5, the corresponding function is manual, back to the origin, single-step, single-cycle, automatic operation.

Three implementation movements of cylinders YG1, YG2, YG3 were by three solenoid YV1, YV2, YV3 controlled. The cylinder YG1 realize moving left and right, the cylinder YG2 realize moving up and down. The cylinders YG3 realize clamping and loosening. The cylinder YG3 has two pistons, intermediate into the oil chamber, left and right cavity of the oil return, left piston to move to the left, right piston shifted to the right, release the part; around into the oil chamber, in the midst of the cavity when the oil back, left piston shifted to the right, right piston to move to the left so as to clamp the part.

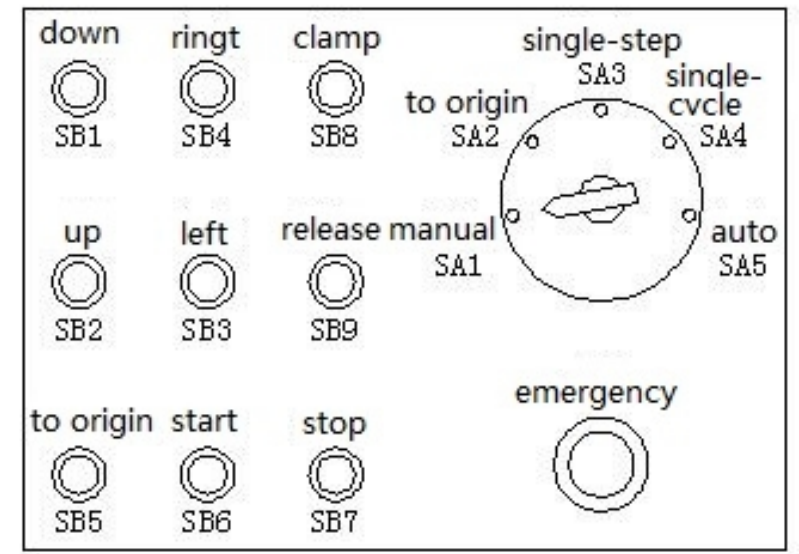

Fig.2 The control panel components layout

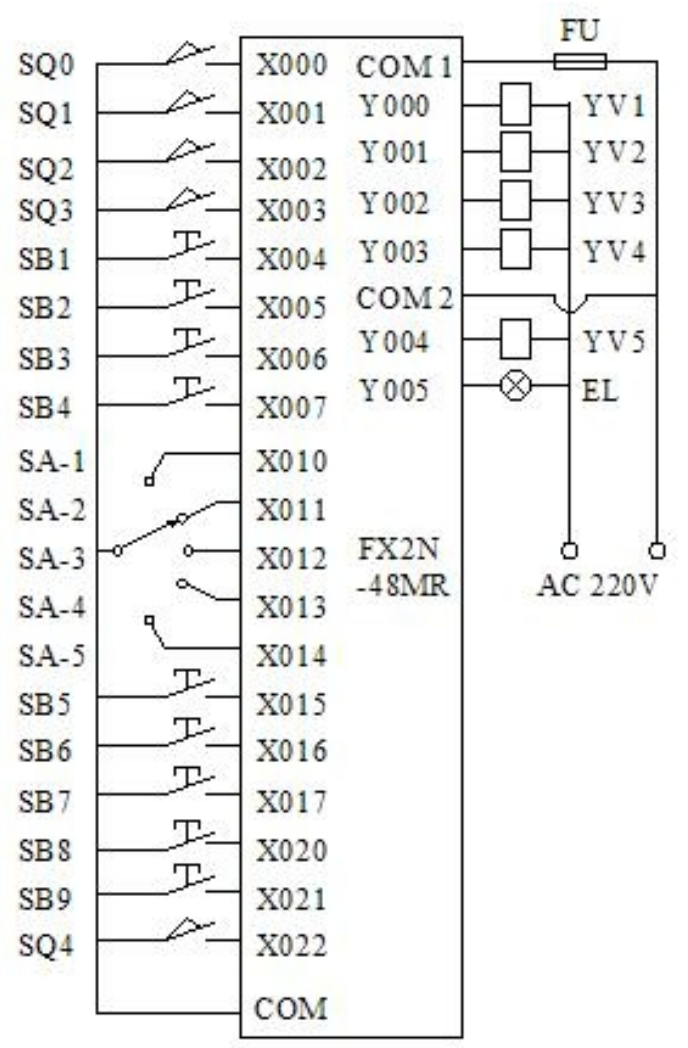

Fig.3 PLC control wiring diagram

For the initialization of the PLC, manual control, automatic back to origin, the program ladder diagram was shown in Fig.4. The Automatic cycle program ladder diagram was shown in Fig.5.

\section{Work Process Analysis}

When turned on, the knob switch turned to the original position SA2, press the homing button SB5, institutions back into the origin, the feeding mechanism is in the leftmost, uppermost. All $\mathrm{s}$ electromagnet YA1, YA2, YA3, YA4, YA5 power off, this state was called in situ. Then the rotary switch turn to the appropriate operating mode, you can start work, the main actions are, the working bodies rise and down, left and right. Clamping device clamp and loosen. When the manual mode, press the corresponding manual button, complete the appropriate action. When the automatic operation mode, press the start button SB6, institutions in accordance with a predetermined program, start automatic work cycle. Automatically work process as follows.

Downward movement and clamping. Select the Auto mode, push the start button SB6, the electromagnet YA2 power on, the solenoid YV2 in the right position, For the cylinder YG2 oil go 
into the upper chamber and the under chamber return, the piston down until press the limit switch SQ1, electromagnet YA2 power off, down moment to stop, while the energized electromagnet YA5 power on, the solenoid YV3 in the left position, For the cylinder YG3 the oil go into the left and right chamber, the intermediate chamber back to the oil, the piston moves to clamp the parts.

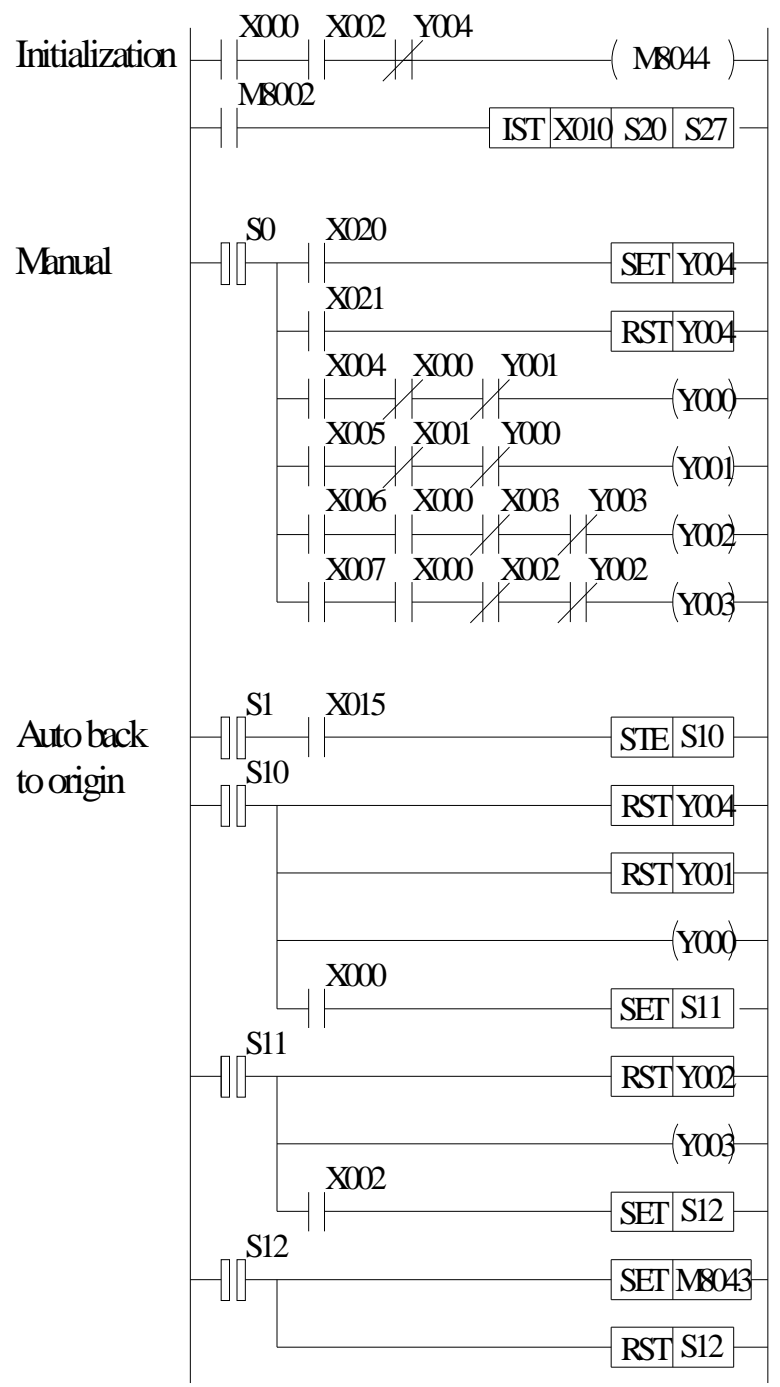

Fig.4 Initialization, Manual, Automatic back to origin ladder diagram

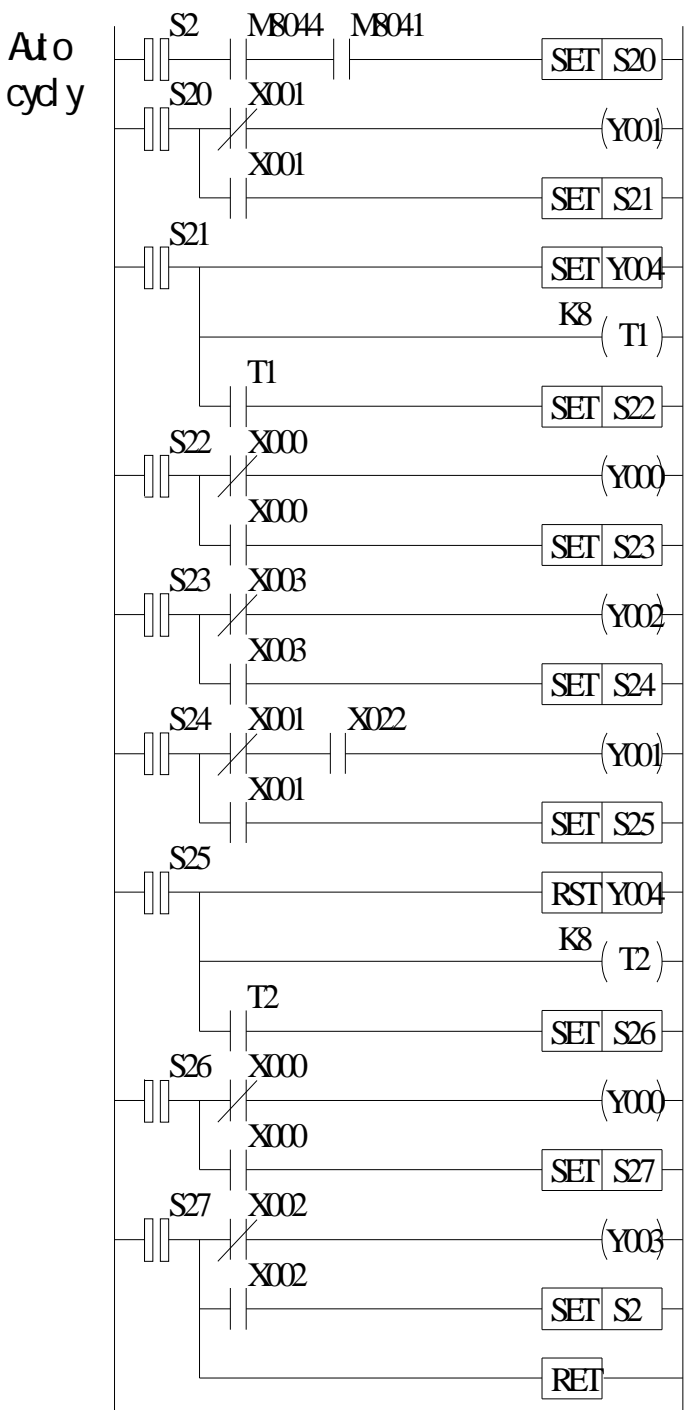

Fig.5 Automatic cycle program ladder diagram

Rise and move to the right. After clamping the parts, the electromagnet YA1 power on, the solenoid YV2 in left position, the cylinder YG2 lower chamber into oil and upper chamber return oil, the piston moves until the pressed limit switch SQ0, the electromagnet YA1 power off, stop rising, while the electromagnet YA3 power on, the solenoid YV1 in the right position, the cylinder YG1 left chamber into oil and right chamber return oil, the piston move to right, until the pressed limit switch SQ3, the electromagnet YA3 power off, right moment stopped.

Downward movement and loosen. Stop right at the same time, the electromagnet YA2 power on, the solenoid YV2 was at right position, the cylinder YG2 upper chamber into oil and the lower chamber back to oil, the piston move down until press the limit switch SQ1, the electromagnet YA2 power off, down moment stop, while the electromagnet YA5 power off, the solenoid YV3 was at right position, the cylinder YG3 intermediate go into oil, left and right chamber return oil, the piston moves, the parts was released.

Up movement and move to the left. After the released the parts, the electromagnet YA1 power on, the solenoid YV2 was at left position, the cylinder YG2 lower chamber into oil and upper chamber return oil, the piston up until pressed limit switch SQ0, the electromagnet YA1 power off, 
the piston stopped rising, while the electromagnet YA4 power on, the solenoid YV1 was at left position, the cylinder YG1 right chamber into oil and left chamber back to oil, the piston moved left until the pressed limit switch SQ2, the electromagnet YA4 power off, the piston stopped left, went back into the origin, completed a cycle of work.

Next, the electromagnet YA2 power on, the solenoid YV2 was at right position, the cylinder YG2 upper chamber into oil and under chamber return oil, the piston moved down, the next working cycle started.

\section{Conclusion}

1) The control program has five working mode, that was manual, back to the origin, single step, single cycle and automation. Convenient for installation and debugging, equipment maintenance.

2) The initialization program used to set up the origin conditions and the initial state, including special auxiliary relay M8044 set origin position, M8002 used as an execution condition of IST. The source element of IST from X10, means X10 X17 has the functions of the PLC wiring diagram.

3) The part detection switch SQ4 was installed on the frame body. To ensure when the feeding mechanism fall to the table, there was no part on the table. To Avoid feeding mechanism collide table.

\section{References}

[1] S.H. Chen: Machinery equipment electrical control (South China University pres, China 2002).

[2] Y.S. Fan: Electrical Control and PLC applications (China Electric Power Press, China 2006) (in Chinese).

[3] Y.J. Jia, D.Y. Zhang, G.Z. Cheng: Applied Mechanics and Materials Vol. 164 (2012) pp 344-347.

[4] X.Q. Chen, G. L. Jiang and G.Z. Cheng: Advanced Materials Research Vol. 568 (2012) pp 43-46

[5] Z.Q. Qi. Machine Electrical Control Technology(Machinery Industry Press, China 2002).

[6] Y.J. Jia and G.Z. Cheng: J. Hydraulic and pneumatic, Sep.2009, pp4-6 (in Chinese).

[7] G.Y. Wang, S.W. Li and G.Z. Cheng: J. Coal Mine Machinery, Aug.2007, pp114-115 (in Chinese).

[8] Y.J. Jia and Y.B.Cheng: Advanced Materials Research Vols. 295-297 (2011) pp 2483-2486.

[9] G.Z.Cheng: Automatic grinding machine electrical control system[J] Manufacturing Technology \& Machine, 2011 (9): 81-83. 